\title{
Die Zukunft hat begonnen
}

\section{Michel Matter}

Dr. med., Vizepräsident der FMH, Departementsverantwortlicher Dienstleistungen und Berufsentwicklung

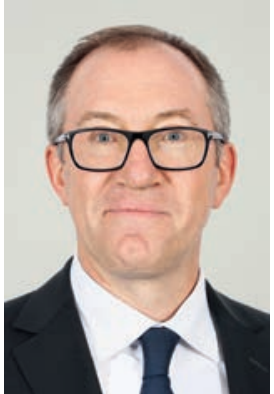

Man sagt, dass die in den 1980er und 1990er Jahren geborene Generation der sogenannten Millennials eine Generation für sich ist, deren gemeinsame Werte auf starken Überzeugungen beruhen und oft im Widerspruch zur heutigen Gesellschaft stehen. Die Normen der Vergangenheit werden so über den Haufen geworfen. Das Gesellschaftsmodell dieser Generation beruht auf der Work-Life-Balance, bei der Arbeit nicht mehr als zentraler Wert des Lebens angesehen wird. Stattdessen bemüht man sich, Privat- und Berufsleben in Einklang zu bringen. Das hat zur Folge, dass ein höherer Anteil dieser Berufstätigen in Teilzeit arbeitet. Dies wiederum bedeutet, dass die Arbeitsbedingungen neu überdacht und angepasst werden müssen. Auch die zunehmende Feminisierung unseres Berufsfelds mit den

\section{Diese Generation erwartet organisatorische Veränderungen, um berufliche Flexibilität zu gewährleisten und neue Ideen umzusetzen.}

damit einhergehenden Auswirkungen unterstreicht den Wunsch nach einer Gesellschaft mit mehr Gleichberechtigung bei der Verteilung von Aufgaben und Berufschancen und die Notwendigkeit, die Gewichtungen an das Weltbild der Millennials anzupassen. Diese wünschen sich ein Arbeitsumfeld, das nicht mehr auf pyramidenförmigen Hierarchien, sondern auf Teamarbeit in effizienten und kooperativen Gruppen basiert. Diese Generation erwartet organisatorische Veränderungen, um berufliche Flexibilität zu gewährleisten und neue Ideen umzusetzen, aber auch konstruktives Feedback und die Bereitschaft, sich immer wieder zu hinterfragen.

Auch messen die Millennials ethischen Werten eine hohe Bedeutung bei. Auf der Grundlage ihrer eigenen Definition von Moral und politischer Korrektheit beäugen sie eine Vielzahl von Aspekten kritisch. Dieses Hinterfragen der Werte eines Unternehmens oder einer Branche führt dazu, dass der Arbeitgeber kontinuierlich Rechenschaft ablegen muss und dass sein Verhalten in den sozialen Netzwerken genau untersucht und diskutiert wird.
Die Welt befindet sich im permanenten Wandel, Neuigkeiten werden umgehend geteilt, die Grenzen zwischen dem Anderswo und dem Zuhause verschwimmen. Auch wird die Definition von Wahrheit permanent in Frage gestellt. Diese Revolution im Hinblick auf Gesellschafts- und Zeitverständnis macht auch vor der Medizin nicht halt. Die Digitalisierung hat für einen ständigen Fluss von Informationen in Echtzeit gesorgt. Die Einführung und das Primat elektronischer Daten werden die derzeitige Arbeitsorganisation völlig verändern. Dabei verringert sich die tatsächliche Zeit, die Patientinnen und Patienten gewidmet wird, drastisch zugunsten einer virtuelleren und stärker vernetzten Vision.

Wir stehen an einem generations- und organisationsbezogenen Scheideweg: Die Zukunft hat begonnen. Auf Vorschlag des Departements Dienstleistungen und Berufsentwicklung hat der Zentralvorstand beschlossen, ab Herbst dieses Jahres eine Umfrage bei den Medizinstudentinnen und -studenten im vierten, fünften und sechsten Studienjahr durchzuführen, um deren Ansprüche an ihre Berufsverbände in Erfahrung zu bringen. Diese Studie wird es der FMH ermöglichen, sich im Hinblick auf die zukünftige Generation von Ärztinnen und Ärzten zu positionieren und ihre Leistungen entsprechend zu optimieren. In einigen angelsächsischen Ländern ist es üblich, Studierende sehr früh in

Es ist von zentraler Bedeutung, die neue Generation besser kennenzulernen und sie auf dem Weg in die Berufswelt begleiten zu können.

ihrem Werdegang in die ärztlichen Vereinigungen aufzunehmen, um für kommende Generationen attraktiv zu sein. Es ist von zentraler Bedeutung, die neue Generation besser kennenzulernen und sie auf dem Weg in die Berufswelt begleiten zu können. Die FMH muss sich dieser Herausforderung stellen und dabei eng mit den Basisorganisationen zusammenarbeiten. 